\title{
Metrizoate Meglumine
}

National Cancer Institute

\section{Source}

National Cancer Institute. Metrizoate Meglumine. NCI Thesaurus. Code C95058.

METRIZOATE MEGLUMINE RJYGJR42WQ 